\title{
Optimal Design of High Power LED Lamp Heat Dissipation Based on Equivalent Thermal Circuit Method
}

\author{
Feng Liang ${ }^{* 1,3, a}$, Lianyu Zhao ${ }^{1, b}$, Youjun Yue ${ }^{2, c}$, Zhuoyin Tong ${ }^{1,3}$, Lizhu Wang ${ }^{1,3}$ \\ ${ }^{1}$ School of mechanical engineering, Tianjin University of Technology, Tianjin 300384, China; \\ ${ }^{2}$ School of automation, Tianjin University of Technology, Tianjin 300384, China; \\ ${ }^{3}$ Tianjin Key Laboratory of the Design and Intelligent Control of the Advanced Mechatronical System, \\ Tianjin 300384, China. \\ aelvislf@outlook.com, blianyuzhao@163.com, cbakeryueyj@163.com \\ Corresponding Author: Feng Liang
}

\begin{abstract}
Keywords: optimal design; micro heat pipe array; high power LED lamp dissipation; equivalent thermal circuit method.
\end{abstract}

\begin{abstract}
Since the heat dissipation problemis harsh in high power LED lamp, in our studywe suggest an optimal design method of high-power LED lamps radiator based on the equivalent thermal circuit method, we abstract mathematical model of the heat dissipation system of the lamps, then chose several key parameters of radiator--spacing of fins $S$, height of fins $H$, thickness of fins $t$ and number of Micro Heat Pipe Array $N_{H}$ as design parameters, and set minimum temperature of the chips as optimization objective. Based on the optimization algorithm we have mentioned, we designed a new radiator for high power LED lamps, through steady-heat-thermal analysis in ANSYS software, the junction temperature is far below the requirement. Therefore, the optimization algorithm can be effectively applied to radiator design of high power LED devices with the advantage of accuracy and swiftness.
\end{abstract}

\section{Introduction}

As the fourth generation of illumination source, high power Light-emitting-diode was highly appreciated by its advantages such as environment friendly, green-power, long lifespan, et al. Nowadays, the heat flux density of high power LED chip has exceeded $100 \mathrm{~W} / \mathrm{cm}^{2}$, whereas limited by package material and other opponents, there are only $10 \%-20 \%$ of input power can transfer to usefully luminous energy, the rest of them dissipate to environment by heat ${ }^{[1]}$. Therefore, as the increasing of input power and lighting brightness, heat dissipation problem has been the major obstacle of popular using of high-power LED lamps.

In order to solve this problem, several domestic and international scholars have done lots of works, they proposed that proper designed heat sink is usually considered to be the best choice economically and effectively ${ }^{[2]}$.As we known, surface area and the conductivity of heat sink aretwo vital parameters influence cooling effective enormously. D. Bhanja researched a structure T-shaped fin with variable thermal conductivity, and found a closed form of analytical solution for analyzing the temperature distribution, performance and optimum design ${ }^{[3]}$. Since higher conductivity materials like copper-aluminum composite structure will be too expensive and heavy for LED lamps, combination of heat pipe and traditional fin radiator is the economically way to compromise. Lu Xiang-you et al improved improve the thermal characteristics of high-power LED package by using a loop heat pipe, and the junction temperature of high-power LED could be controlled steadily under $100^{\circ} \mathrm{C}$ for the heat load of $100 \mathrm{~W}^{[4]}$. Lü Xiao investigated the effect of groove size on heat transfer performance of a loop heat pipe(LHP) with flat evaporator using experimental method, then found the heat transfer performance and thermal resistance decrease with increasing heat load and then level off for the two different grooves ${ }^{[5]}$.

In this paper, we set several micro heat pipe arrays(MHPAs) ${ }^{[6][7]}$ in traditional fin heat sink in 
order to increase thermal conductivity, then we suggest an optimal method of this unique heat-sink based on equivalent thermal circuit method, choose spacing of fins $S$, height of fins $H$, thickness of fins $t$ and number of Micro Heat Pipe Array $N_{H}$ as design parameters, and we set minimum junction temperature of the chips as optimization objective. A simplified 3-D model of high power LED lamp equip with the new heat-sink is built up, then we use FEM software ANSYS to conduct a steady state thermal simulation, the results show that the new heat-sink assembled MHPAs gets the ability to solve the heat dissipation problem of high power LED lamps.

\section{Optimal design of heat sink of high power based on equivalent thermal circuit method}

\subsection{Physical model establishment}

As the objective of this paper, the main structures of high power LED lamp including: lamp housing, holder, transparent cover, reflective plate, LED arrays, MCPCB, power supplier and aluminum fin radiator et al, represented in figure 1 . We just consider parts have straightly influence on thermal dissipation for simplicity, separately, LED arrays, MHPAs, MCPCB and aluminum fin radiator are key parts of our thermal model.

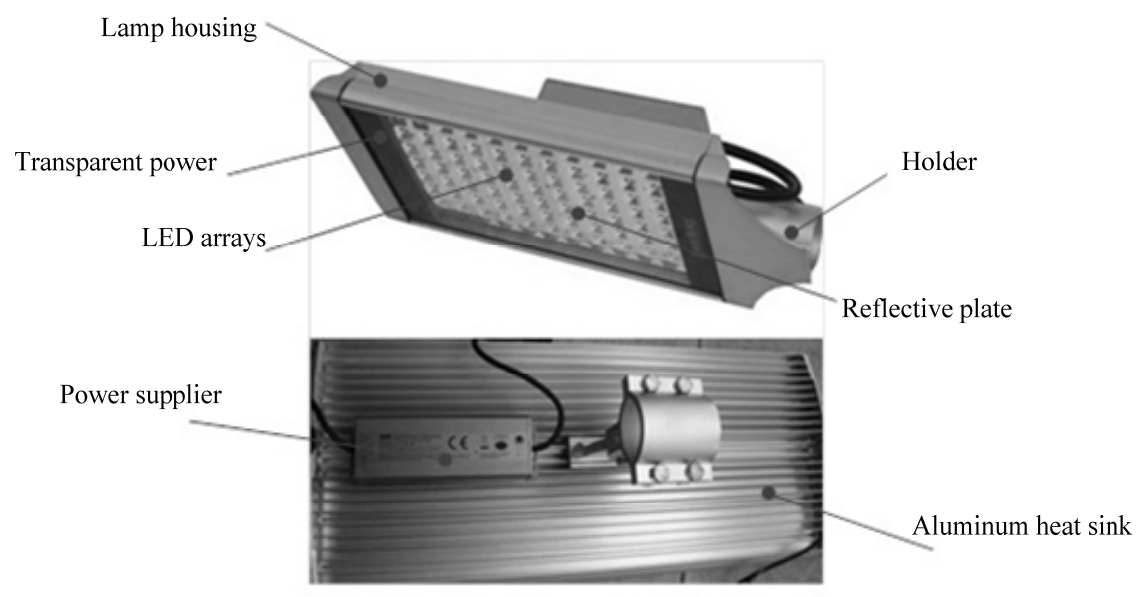

Fig. 1 Main structure of LED lamp

In order to build thermal dissipation model, we should simplify the 3-D construction showed in figure 1, according to several hypothesis we made:

(1).Input power of each LED lamp bead is constant;

(2).Each construction we discuss here is material uniformity and has constant heat conductivity;

(3). The natural convection environment is in dry air at standard atmospheric pressure, and air temperature $\mathrm{T}_{\mathrm{A}}$ is constant;

(4). The natural convection coefficient $\alpha$ is only related to fin temperature $\mathrm{T}_{\mathrm{F}}$;

(5).Since the thickness of fint is far smaller than the height of fin $\mathrm{H}$, therefore we ignore influence of end face of fin during heat convection;

(6).Neglect the radiation of heat sink, only consider the heat conduction and convection. 


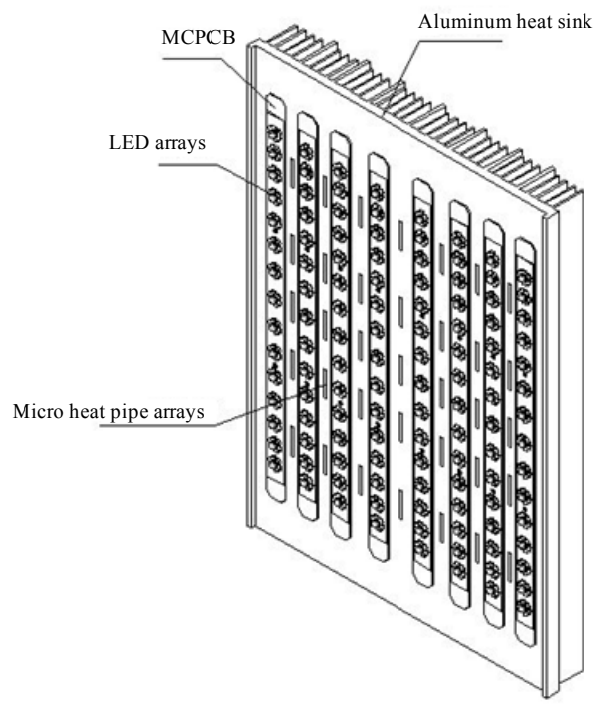

Fig. 2Simplified thermal dissipation model of high power LED lamp

Figure 2 shows simplified physical model we have built, there are several MHPAs spacing in the base of fin heat sink, as we mentioned before, they can sharply increase the heat conductivity of the radiator.

\subsection{Construction of mathematical model of the heat dissipation system}

After above analysis and simplify, the dissipation problem of high power LED lamp has simplified to one dimensional steady heat transfer problem, furthermore, we transform heat transfer path to equivalent thermal circuit form. Based on the physical model, we analysis the heat transfer path of the model and then illustrate thermal resistance network diagram in figure3.

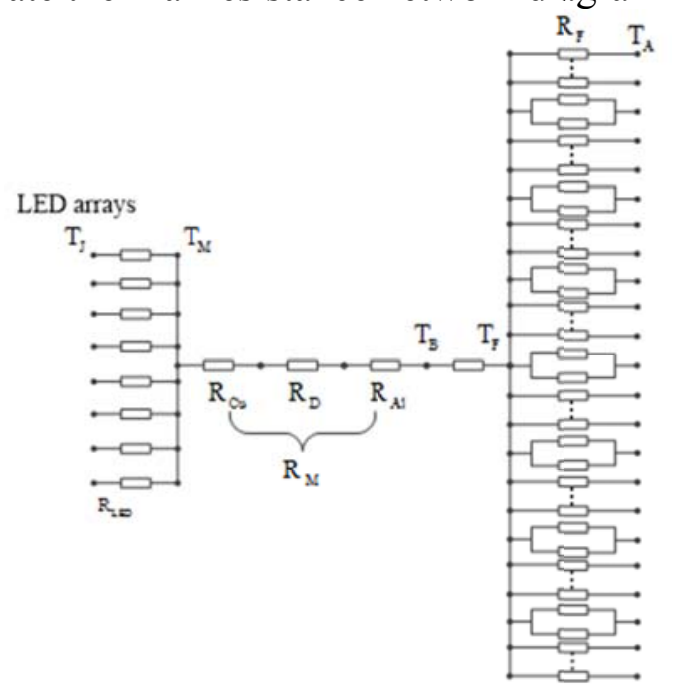

Fig. 3Thermal resistance network diagram

In order to determine parameters of the figure 3, based on the series-parallel connection of thermal network, we can establish mathematical model of thermal dissipation as followed:

$$
R_{J-A}=\frac{R_{L E D}}{N_{L E D}}+R_{C u}+R_{D}+R_{A l}+R_{B}+R_{F}=\frac{T_{J}-T_{M}}{Q_{T}}+\frac{T_{M}-T_{B}}{Q_{T}}+\frac{T_{B}-T_{F}}{Q_{T}}+\frac{T_{F}-T_{A}}{Q_{T}}
$$

In formula(1), $\mathrm{R}_{\mathrm{LED}}$ is the package resistance of LED lamp beaded, could be found in the guide books or the instruction $\mathrm{s}$ of products, and $\mathrm{N}_{\mathrm{LED}}$ is the number of lamps, $\mathrm{R}_{\mathrm{Cu}}$ is the resistance of the copper layer of $\mathrm{MCPCB}, \mathrm{R}_{\mathrm{D}}$ is the resistance of the dielectric layer of $\mathrm{MCPCB}, \mathrm{R}_{\mathrm{Al}}$ is the resistance of the Aluminum grassroots of $\mathrm{MCPCB}$ and $\mathrm{R}_{\mathrm{F}}$ stand for the resistance of fin part of new heat sink.

Since $\mathrm{R}_{\mathrm{LED}}$ and $\mathrm{N}_{\mathrm{LED}}$ we have already known, $\mathrm{R}_{\mathrm{Cu}}, \mathrm{R}_{\mathrm{D}}$ and $\mathrm{R}_{\mathrm{Al}}$ could be calculated in the following 
formula(2).

$$
\mathrm{R}_{X}=\frac{\delta_{X}}{\lambda_{X} \mathrm{~A}_{X}}=\frac{\delta_{X}}{\lambda_{X}\left(\mathrm{f} \cdot \mathrm{L}_{X} \cdot \mathrm{H}_{X}\right)}
$$

Wherein, $X$ representative copper layer $\mathrm{C}_{\mathrm{u}}$, dielectric layer $\mathrm{D}$, aluminum grassroots $\mathrm{A}_{1}$ and aluminum base plate of heat sink B separately; $\delta_{X}$ is the thermal conductivity coefficient of materials layers, $A_{X}$ is the area for the heat flow along the vertical direction, $f$ is the surface coefficient, for the cooper layer $\mathrm{f}=0.8$ and all other surface $\mathrm{f}=1$.

Based on the thermal resistance formula(1) and the thermal resistance network, we could deduced that resistance $\mathrm{R}_{\mathrm{F}}$ of heat sink is consisted of two different parts, one is traditional rectangular section fins resistance $\mathrm{R}_{\mathrm{F}}$ and the other is micro heat pipe array resistance $\mathrm{R}_{\mathrm{H}}$, however $\mathrm{R}_{\mathrm{H}}$ usually given in the instruction of MHPAs.

And resistance $\mathrm{R}_{\mathrm{F}}$ ' could be decided by the following formula:

$$
\mathrm{R}_{\mathrm{F}}^{\prime}=\frac{1}{\alpha \mathrm{A} \eta_{\mathrm{F}}}=\frac{1}{\alpha \eta_{\mathrm{F}}\left(2 \mathrm{~N}_{\mathrm{F}} \mathrm{H}_{\mathrm{B}} \mathrm{W}\right)}
$$

While $\mathrm{A}$ is the total non-ignored surface area vertical to the heat flow direction, $\eta_{\mathrm{F}}$ is the fin efficiency that could be determined empirically.

From the thermal resistance network, it is easy to find that two parts of thermal resistance is paralleled to each other, therefore, the total thermal resistance $R_{F}$ could be determined as followed:

Thus,

$$
\frac{1}{\mathrm{R}_{\mathrm{F}}}=\frac{1}{\mathrm{R}_{\mathrm{F}}{ }^{\prime}}+\frac{\mathrm{N}_{\mathrm{H}}}{\mathrm{R}_{\mathrm{H}}}
$$

$$
R_{F}=\frac{R_{F}^{\prime} \cdot R_{H}}{R_{F}^{\prime} \cdot N_{H}+R_{H}}
$$

\section{3 calculation process}

Based on all above discussion, we suggest an optimal method for determine key parameters of the new heat sink with MHPAs. For convenience of specific calculation, we chose a typical $180 \mathrm{~W}$ LED lamp product in the market, and redesigned the heat sink with method we suggested. Considering both practically and easily machining, we set base size of the new heat sink to length is $\mathrm{L}_{\mathrm{B}}=500 \mathrm{~mm}$, width is $\mathrm{H}_{\mathrm{B}}=360 \mathrm{~mm}$ and thickness is $\delta_{\mathrm{B}}=10 \mathrm{~mm}$, then chose spacing of fins $S$, height of fins $H$, thickness of fins $t$ and number of Micro Heat Pipe Array $N_{H}$ as design parameters, and we made sure minimum junction temperature of the chips satisfied to $\mathrm{T}_{\mathrm{J}} \leq 70{ }^{\circ} \mathrm{C}$. We give the initials of iteration calculation as following: spacing of fins $S=8 \mathrm{~mm}$, height of fins $H=36 \mathrm{~mm}$, thickness of fins $t=2 \mathrm{~mm}$ and number of Micro Heat Pipe Arrays $N_{H}=34$.

Before calculation process, we should give calculation parameters in formula (1)---(5), input power of each LED lamp beaded is constantly $1.5 \mathrm{~W}$, consider photoelectric efficiency as $15 \%$, the total heat generation of the all 120 lamps beaded is $\mathrm{Q}_{\mathrm{T}}=102 \mathrm{~W}$. 


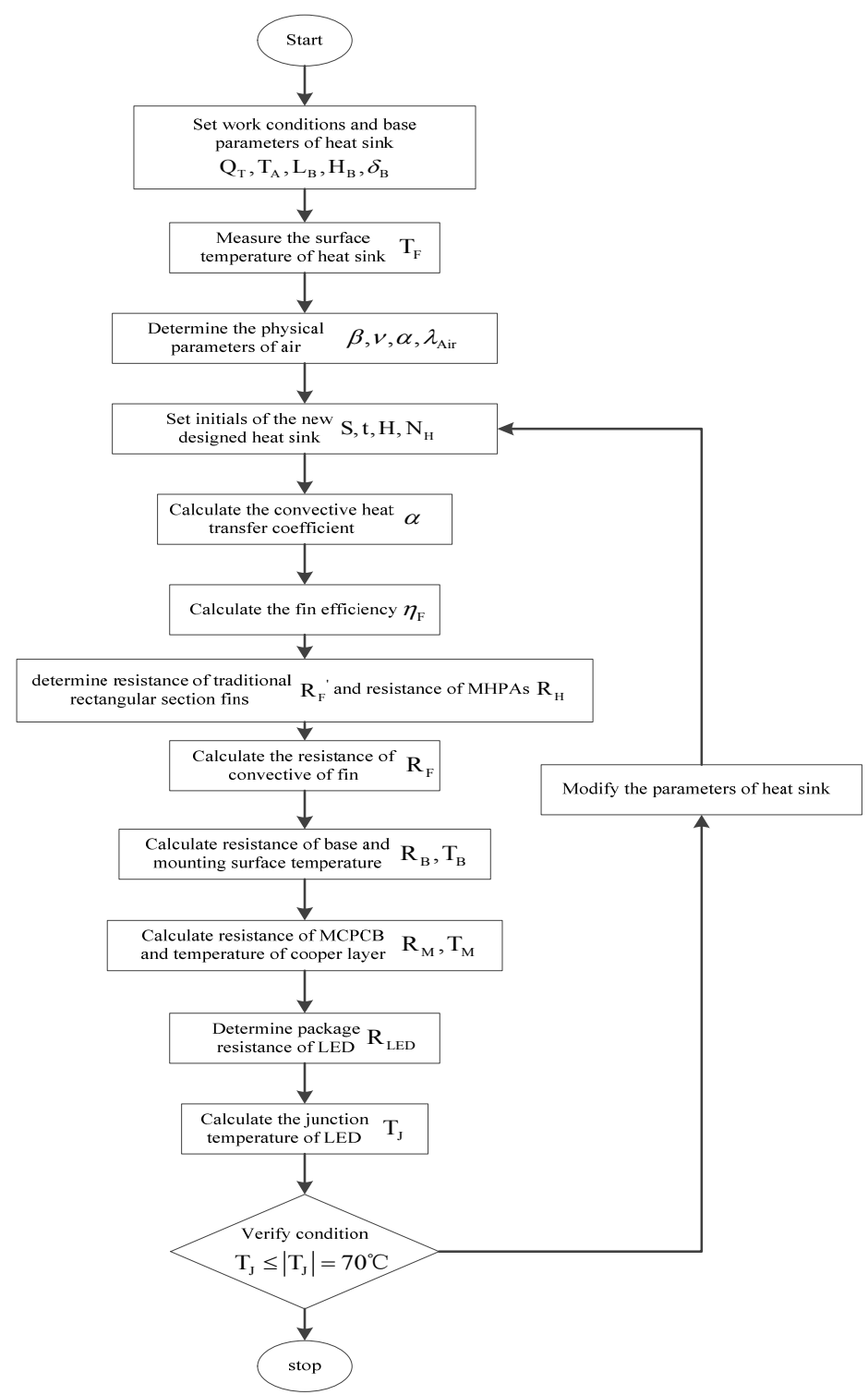

Fig. 5 Calculation flowchart

For the convenience of programming calculation, we draw the flowchart in figure 5, then we could program with MATLAB or other software to calculate the parameters to meet the requirements, we use MATLAB to solve the problem and get a group of optimal parameters that we expected. According to our calculated results, we get a group of optimal parameters, spacing of fins $S=6.5 \mathrm{~mm}$, height of fins $H=36 \mathrm{~mm}$, thickness of fins $t=1.8 \mathrm{~mm}$ and number of Micro Heat Pipe Arrays $N_{H}=35$.

\section{Finite Element Method verification}

Under normal circumstance, we can verify the validity of the new designed heat sink with ether experimental or numerical simulation methods, since the numerical simulation method have commonly applied in engineering practice, and considering the high cost and uncertainty of the results of physical experiment, we chose numerical simulation method FEM to demonstrate the effectiveness of the new heat sink of high power LED lamp.

\subsection{Modelling and pre-treatmentFEM}

We complete the steady thermal simulation in ANSYS Workbench, since we have finished 3-D assembly model of high power LED lamp in Solidworks, including heat sink. Considering the symmetry of the model, we introduce the symmetry boundary condition in order to simplify the model, and before analysis, we also idealize the model to improve computation accuracy and reduce the waste of computation resource. 
The key step of FEM is the discretization of assembly model, we chose structural grid for its superiority in fluid and heat transfer simulation. For different spare parts, we chose different mesh option to control grid quantity and quality.

However, it would be extremely complex to simulate the phrase change and heat transfer process inside the micro heat pipe accurately, so we considerthe micro heat pipe arrays asisotropous solid rods of constant conductivity $\mathrm{K}=2500 \mathrm{~W} / \mathrm{m} \cdot \mathrm{K}$, while $\mathrm{K}$ is a value derived from empirical formula and experimental works ${ }^{[8]}$. Other related thermal parameters of the materials during the simulation that are given in tab.1 as followed.

Table1 thermal parameters of the materials

\begin{tabular}{cccc}
\hline Materials & Thermal conductivity & Specific heat & Density \\
& $\mathrm{W} / \mathrm{m} \cdot \mathrm{K}$ & $\mathrm{J} /\left(\mathrm{kg} \cdot{ }^{\circ} \mathrm{C}\right)$ & $\mathrm{kg} / \mathrm{m}^{3}$ \\
\hline Sappire & 57 & 300 & 2400 \\
PPA & 0.077 & 1256 & 1300 \\
Epoxy & 0.12 & 855 & 1252 \\
MCPCB & 2 & 800 & 2700 \\
Heat sink & 401 & 385 & 8300 \\
Aluminum alloy & 237.5 & 951 & 2689 \\
Copper alloy & 400 & 385 & 8933 \\
\hline
\end{tabular}

We set the boundary condition of the simulation as the third boundary condition, and set LED chips as internal heat generator to simulate the constant input power of the chips. The computation type is steady thermal simulation as we mentioned before, and the ambient temperature is $25^{\circ} \mathrm{C}$. In order to speed the convergence of calculation, we neglect the contact thermal resistance of the spare parts of the assemblies, and we defined the contact type of those spare parts as MPC in WORKBENCH.

\subsection{FEM simulation results}

From the temperature contours in figure 8 , we can easily found that the highest temperature is $52.503^{\circ} \mathrm{C}$, located in the middle of the LED arrays. In order to illustrate the dissipation enhancement of the new designed heat sink equipped with MHPAs, we modeled a heat sink without MHPAs, whereas other parameters are totally the same as the former one, then we simulated the dissipation effect under the same setting. Compared with the contrast heat sink, we notice that the high power of heat sink without MHPAs is $77.335^{\circ} \mathrm{C}$, it is much higher than our new designed heat sink, and the distribution of the temperature is more uniform in the high power LED lamp equipped with the new one.

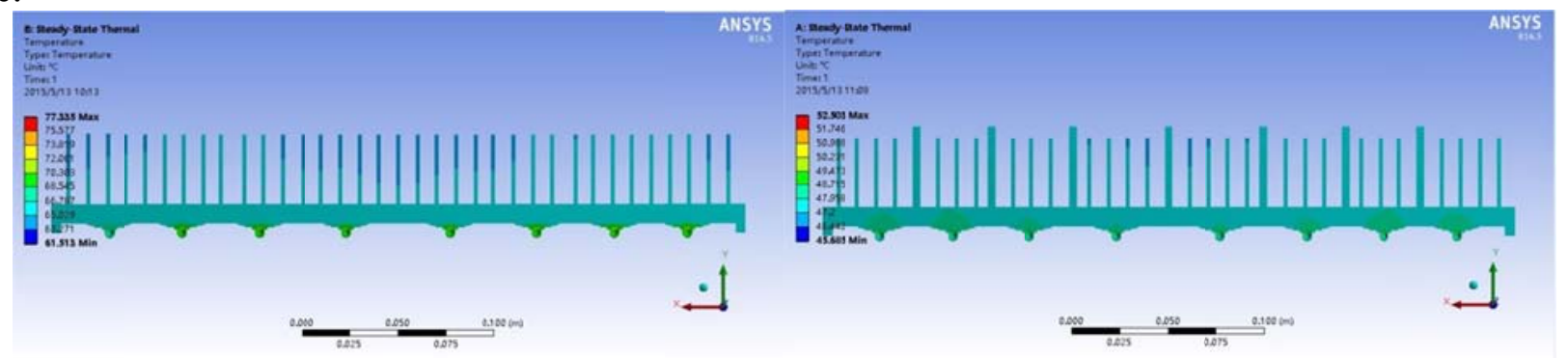

a. Heat sink without MHPAs b. heat sink with MHPAs

Fig. 6Temperature contour comparison of heat sink with and without MHPAs

\section{Conclusions}

Our new designed heat sink involves four major parameters,spacing of fins $S$, height of fins $H$, thickness of fins $t$ and number of Micro Heat Pipe Arrays $N_{H}$, for the optimal performance, we suggest an optimal algorithm based on equivalent thermal circuit method, and we determined these parameters byprogramming in MATLAB。 
Form the simulation model and the contrast model, we found the highest junction temperature of LED lamp equip with new designed heat sink is $52.503^{\circ} \mathrm{C}$ while the LED lamp equip with the traditional one is $77.335^{\circ} \mathrm{C}$, so the heat sink with MHPA inside got the ability to improve the thermal uniformity, and its enhancement of thermal dissipation is apparently. All in all, the new designed heat sink characterized by the optimal algorithm we suggest could deal with thermal dissipation problem of high power LED lamp effectively.

\section{Acknowledgement}

Tianjin Science and Technology Project: 132ZCZDGX03200.

\section{Reference}

[1]. ClmensJ. M. Lasance, András Poppe, Cathy Biber. Thermal management for LED applications[B]. Springer express, 2014. P53

[2]. Shih-Jeh Wu, Shih-Chen Hsu, Shen-Li Fu, et al. Numerical simulation of high power LED heat dissipation system[J].Electron mater lett. Vol.10, No.2(2014), P497-502

[3]. D. Bhanjar, B. Kundu. Radiation effect on optimum design analysis of a constructal T-shaped fin with variable thermal conductivity[J]. Heatmass transfer. Vol.48, (2012), P109-122

[4]. Xiang-you Lu, Tse-Chao Hua, Mei-Jing Liu. Thermal analysis of loop heat pipe used for high-power LED[J]. Thermal acta. Vol.493, (2009), P25-29

[5]. Xiao Lü, Jia-jin Wei, Yan Long. Effective of groove size on heat transfer performance of loop heat pipe with flat evaporator [J]. Journal of engineering of thermophysics. Vol.36, No.3(2015), P611-614

[6]. Yao-hua Zhao, Hong-yan Wang, Yan-hua Diao, et al. Heat transfer characteristics of flat micro heat-pipe array[J]. CIESC Journal. Vol.62, No.2(2011), P336-343

[7]. Hong-yan Wang, Yao-hua Zhao, Li-min Hao, et al. Applied investigation on LED heat cooling equipment using flat micro-heat pipe arrays[J]. Semiconductor technology. Vol.37, No.3(2012), P240-244

[8]. H. Mroue, J.B. Ramos, L.C. Wrobel et al. Experimental and numerical investigation of an air-to-water heat pipe-based heat exchanger[J]. Applied thermal engineering. Vol.78, No.5(2015), P339-350 